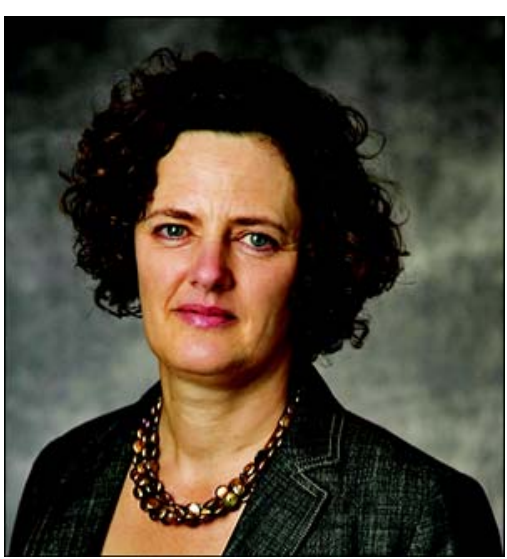

Kath M. Bogie, DPhil

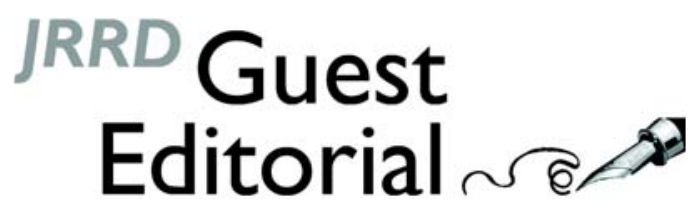

\title{
Evidence-based practice in wound care: Toward addressing our knowledge gaps
}

\section{BACKGROUND}

Chronic nonhealing wounds are a major complication for many veterans. Particularly at risk are veterans with reduced mobility, such as those who have suffered a spinal cord injury (SCI). Chronic wounds cause significant suffering, including profound negative effects on general physical health, socialization, financial status, body image, level of independence, and control. For individuals with SCI, the development of a pressure ulcer is one of the leading causes of readmission to the hospital after acute rehabilitation and a major source of morbidity and even mortality. The management of chronic wounds is also extremely costly for clinical facilities, from the acute care setting to long-term care.

Many clinical practice guidelines (CPGs) for wound care are currently being issued with the overall goal of reducing the incidence and prevalence of this significant healthcare complication. CPGs have the potential to improve the standard of care for chronic wounds and decrease associated costs. A wealth of basic science and early clinical trials are being carried out in the field of chronic wound care research. However, there remains a disconnect between early stage research efforts and implementation as routine clinical practice in the care of veterans with chronic wounds.

The 2nd International Conference on Evidence Based Practice in Wound Care: The Effective Implementation of Pressure Ulcer Clinical Practice Guidelines was held in Cleveland, Ohio, in June 2009 [1]. This program was designed for the many specialties involved in the interdisciplinary field of wound care research. The focus of the conference was on topics related to the effective selection and implementation of evidence-based CPGs. Over 150 attendees were provided with educational tools to enable them to effectively implement evidence-based practice approaches to pressure ulcer care. While many felt that they were familiar with CPGs for their specialty, there were concerns that implementation could be hindered by lack of support and continuing education in best evidence-based practices for wound care.

In conjunction with the 2nd International Conference on Evidence Based Practice in Wound Care, an expert panel was convened in June 2009 to develop a research agenda based on critical knowledge gaps regarding pressure ulcers in individuals with SCI and on the implementation of advanced clinical practices. A literature-based discussion of the consensus panel conclusions is presented as a second Guest Editorial in this issue of JRRD [2]. The research articles presented illustrate both preclinical and clinical research that will lead to improved rehabilitative and lifetime outcomes for at-risk veterans, particularly those with SCI. 


\section{ARTICLES IN THIS ISSUE}

"Effect of sensory and motor electrical stimulation in vascular endothelial growth factor expression of muscle and skin in full thickness wound." Asadi et al.

Electrotherapy for the treatment of chronic wounds has long been known, and there have been many clinical reports conducted on the technique. However, widespread implementation has been limited by lack of definitive proof demonstrating the positive effects of electrical stimulation (ES) on wound healing. The current limitations imposed on electrotherapy usage by the Centers for Medicare and Medicaid Services and the lack of Food and Drug Administration approval reflect the underlying deficit in understanding of the physiological mechanism that is an essential precursor to optimization of clinical therapy. In their article, "Effect of sensory and motor electrical stimulation in vascular endothelial growth factor expression of muscle and skin in full thickness wound," Asadi et al. report on a preclinical study employing an acute animal wound model [3]. Varying ES paradigms were employed to study the effect on angiogenesis, as indicated by expression of vascular endothelial growth factor (VEGF). The authors found significant differences between the effects of sensory and motor ES. In this acute full-thickness wound model, sensory ES produced significantly higher levels of VEGF in skin wound tissue at 1 week postinjury, compared with both control and motor ES. No differences were found in muscle tissue underlying the wound. The authors propose that sensory ES may facilitate cell migration, as indicated by sustained up-regulation of VEGF. As noted by the authors, the mechanisms by which sensory ES up-regulates VEGF in skin wound tissue remain to be elucidated. The current article is a valuable contribution toward understanding the physiological mechanisms underlying electrotherapy, a necessary foundation for the implementation of a reliable and effective clinical treatment for chronic wounds.

\section{“Assessing evidence supporting redistribution of pressure for pressure ulcer prevention.” Sprigle and Sonenblum}

Sprigle and Sonenblum's article, “Assessing evidence supporting redistribution of pressure for pressure ulcer prevention,” presents a comprehensive review of preclinical and clinical studies [4]. It is well recognized that pressure ulcer development involves multiple risk factors. A pressure ulcer will occur as a consequence of load applied to the tissue above a specific threshold for a specific length of time. The authors review evidence to support addressing both magnitude and duration of loading in order to provide effective pressure ulcer prevention. Preclinical and clinical studies are considered. Research has shown that tissue tolerance does not have a universal safe loading threshold. The authors address the rationale behind several common clinical interventions and show that the evidence bases for some standard clinical practices are limited. For example, there is limited evidence that a uniform 2-hour frequency is optimal for all at-risk individuals. There is, however, evidence that turning schedules should depend on both the patient's condition and the support surface. Similarly, current guidelines suggest that frequent weight shifting maneuvers are essential for all wheelchair users. Recommendations are based primarily on the SCI population, but still vary greatly. It has recently been found that no direct correlation between frequency of weight shifting and pressure ulcer incidence is evident. Further research is needed to determine optimal turning intervals for patients in bed and weight-shifting recommendations for wheelchair users. This comprehensive review article reinforces the need for evidence-based individualized risk management incorporating personal risk factors.

\section{"Comparison of in-person and digital photograph assessment of stage III and IV pressure ulcers among veterans with spinal cord injuries." Terris et al.}

The use of digital images to monitor wound healing can be a valuable component of a wound care telemedicine clinic and has the potential to improve standard of care in the community. Terris et al. report on a clinical study to evaluate assessment agreement 
when severe pressure ulcers are evaluated in-person and using digital photography. A standardized wound assessment and photography protocol was employed to evaluate pressure ulcers in a group of inpatients with SCI. In their article, "Comparison of in-person and digital photograph assessment of stage III and IV pressure ulcers among veterans with spinal cord injuries," the authors found moderate interrater agreement for both methods [5]. It would appear that, even with a structured evaluation protocol, in-person assessment of severe pressure ulcers is limited, with only 25 percent of wound description categories demonstrating moderate or better agreement. Undermining of the wound margin was most reliably identified by all raters. The authors consider that subjective perceptions of qualitative wound characteristics may be the primary source of interrater variation. At first consideration, this article may appear to provide counterintuitive findings, in that neither assessment method appears to be highly reliable. This may seem to have minimal relevance to evidencebased care. However, it is critically important to determine what does not work well so that improvements can be developed. Subjective perception is always involved in qualitative evaluation of a wound regardless of the method of viewing. The evidence indicates that a standardized wound assessment methodology based on quantitative measures is required.

\section{"Interface shear and pressure characteristics of wheelchair seat cushions.” Akins et al.}

Shear stress is widely recognized as a risk factor in pressure ulcer development. Several recent wheelchair cushion designs have incorporated technologies to reduce interface shear stresses. However, reliable and effective techniques to evaluate shear stresses have not been implemented. In the article "Interface shear and pressure characteristics of wheelchair seat cushions,” Akins et al. report on a preclinical study of 21 commercially available wheelchair cushions [6]. The authors have developed a new methodology to quantify interface shear stress and pressure together with horizontal stiffness. Their findings suggest that the International Organization for Standardization (ISO) 16840-2 horizontal stiffness measure does not provide a complete picture of clinically relevant shear charac- teristics. Concurrent use of a combined interface pressure and shear force sensor in addition to ISO standard testing is recommended for comprehensive assessment of wheelchair cushion shear characteristics. This article represents an important contribution toward standardization of wheelchair cushion characterization, an essential precursor to the development of evidencebased guidelines for seating prescriptions.

\section{CONCLUSIONS}

Pressure ulcers remain a significant complication for many veterans with limited or restricted mobility, particularly those with SCI and the elderly. The overall population at risk for pressure ulcer development is increasing due to changing demographics, and is even more prevalent in the veteran population, with many aging veterans from previous conflicts and increasing numbers of severely injured individuals from Operation Iraqi Freedom/Operation Enduring Freedom. The Veterans Health Research and Development Assessment Program has recognized the need to develop and implement interventions that will lessen the incidence of pressure ulcer development in the veteran population. The articles presented in this single-topic section address various aspects of the current gaps in evidence-based knowledge of wound management, particularly pressure ulcer care.

\section{Kath M. Bogie, DPhil}

Senior Research Scientist, Cleveland Advanced Platform Technology Center and Functional Electrical Stimulation Center, Louis Stokes Cleveland Department of Veterans Affairs Medical Center, Cleveland, OH; Assistant Professor, Departments of Orthopaedics and Biomedical Engineering, Case Western Reserve University, Cleveland, $\mathrm{OH}$

\section{Email: kmb3@case.edu}

\section{REFERENCES}

1. 2nd International Conference on Evidence Based Practice in Wound Care: The Effective Implementation of Pressure Ulcer Clinical Practice Guidelines. 
Cleveland, OH: Case Western Reserve University; 2009 Jun.

2. Henzel MK, Bogie KM, Guihan M, Ho CH. Pressure ulcer management and research priorities for patients with spinal cord injury: Consensus opinion from SCI QUERI Expert Panel on Pressure Ulcer Research Implementation. J Rehabil Res Dev. 2011;48(3):xi-xxxii. DOI:10.1682/JRRD.2011.01.0011

3. Asadi MR, Torkaman G, Hedayati M. Effect of sensory and motor electrical stimulation in vascular endothelial growth factor expression of muscle and skin in full-thickness wound. J Rehabil Res Dev. 2011;48(3):195-202.

DOI:10.1682/JRRD.2009.11.0182

4. Sprigle S, Sonenblum S. Assessing evidence supporting redistribution of pressure for pressure ulcer prevention: A Review. J Rehabil Res Dev. 2011;48(3): 213-24. DOI:10.1682/JRRD.2010.05.0102
5. Terris DD, Woo C, Jarczok MN, Ho CH. Comparison of in-person and digital photograph assessment of stage III and IV pressure ulcers among veterans with spinal cord injuries. J Rehabil Res Dev. 2011;48(3): 225-34. DOI:10.1682/JRRD.2010.03.0036

6. Akins JS, Karg PE, Brienza DM. Interface shear and pressure characteristics of wheelchair seat cushions. J Rehabil Res Dev. 2011;48(3):203-12.

DOI:10.1682/JRRD.2009.09.0145

This article and any supplementary material should be cited as follows:

Bogie KM. Evidence-based practice in wound care: Toward addressing our knowledge gaps. J Rehabil Res Dev. 2011;48(3):vii-X.

DOI:10.1682/JRRD.2011.01.0010

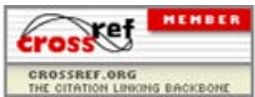

